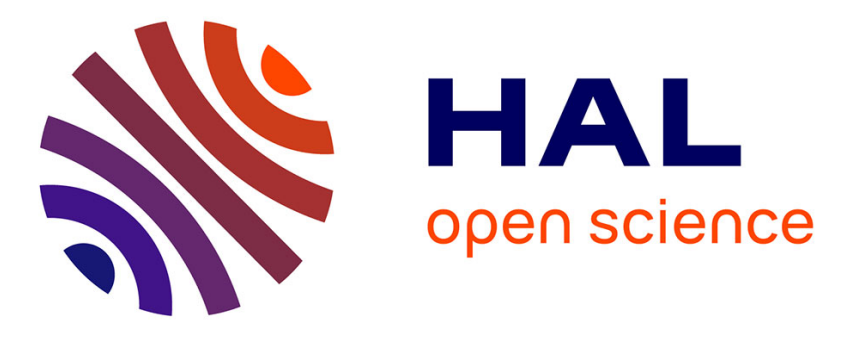

\title{
Computation of electron energy loss spectra by an iterative method
}

Peter Koval, Mathias Per Ljungberg, Dietrich Foerster, Daniel Sánchez-Portal

\section{To cite this version:}

Peter Koval, Mathias Per Ljungberg, Dietrich Foerster, Daniel Sánchez-Portal. Computation of electron energy loss spectra by an iterative method. Nuclear Instruments and Methods in Physics Research Section B: Beam Interactions with Materials and Atoms, 2015, 354, pp.216-219. 10.1016/j.nimb.2014.11.080 . hal-01174984

\section{HAL Id: hal-01174984 https://hal.science/hal-01174984}

Submitted on $10 \mathrm{Jul} 2015$

HAL is a multi-disciplinary open access archive for the deposit and dissemination of scientific research documents, whether they are published or not. The documents may come from teaching and research institutions in France or abroad, or from public or private research centers.
L'archive ouverte pluridisciplinaire HAL, est destinée au dépôt et à la diffusion de documents scientifiques de niveau recherche, publiés ou non, émanant des établissements d'enseignement et de recherche français ou étrangers, des laboratoires publics ou privés.

\section{(ㅇ)(1) $\$$}

Distributed under a Creative Commons Attribution - NonCommercial - NoDerivatives $\mid 4.0$ 


\title{
Computation of electron energy loss spectra by an iterative method
}

\author{
Peter Koval $^{\mathrm{a}, \mathrm{b}, *}$, Mathias Per Ljungberg ${ }^{\mathrm{a}}$, Dietrich Foerster $^{\mathrm{c}}$, Daniel Sánchez-Portal ${ }^{\mathrm{a}, \mathrm{b}}$ \\ ${ }^{a}$ Donostia International Physics Center (DIPC), Paseo Manuel de Lardizabal 4, E-20018 San Sebastián, Spain \\ ${ }^{\mathrm{b}}$ Centro de Física de Materiales CFM-MPC, Centro Mixto CSIC-UPV/EHU, Paseo Manuel de Lardizabal 5, E-20018 San Sebastián, Spain \\ ${ }^{\mathrm{c}}$ LOMA, Université de Bordeaux 1, 351 Cours de la Liberation, 33405 Talence, France
}

Keywords:

Product basis set

Localized functions

Extended systems

Iterative TDDFT

\begin{abstract}
A B S T R A C T
A method is presented to compute the dielectric function for extended systems using linear response time dependent density functional theory. Localized basis functions with finite support are used to expand both eigenstates and response functions. The electron energy loss function is directly obtained by an iterative Krylov subspace method. We apply our method to graphene and silicon and compare it to plane wave based approaches. Finally, we compute electron energy loss spectrum of $C_{60}$ crystal to demonstrate the merits of the method for molecular crystals, where it will be most competitive.
\end{abstract}

\section{Introduction}

The dielectric function (DF) describes the linear response of a solid to external electromagnetic fields $[1,2]$ and so contains infor mation about experimentally observable quantities like optical absorption spectra and electron energy loss spectra (EELS) [3]. Computing the DF $a b$ initio can be efficiently done within time dependent density function theory (TDDFT). Most approaches in the literature employ plane wave (PW) basis sets [4 8], although several alternatives that use atomic orbital basis sets have been published [9 11]. PWs offer a natural framework for solids, a stable convergence with the basis set size and diagonal representation of some of the relevant operators. On the other hand, the linear com bination of atomic orbitals (LCAO) method allows for an economi cal description of the electronic structure, especially for molecular solids and open nano structures. In this work, we present a method that consistently uses basis sets of finite support both in the initial density functional theory (DFT) and in the subsequent TDDFT cal culation. We directly obtain the electron energy loss function by using an iterative method, thus avoiding costly matrix inversions. We start the derivation from widely used PW formulas to make apparent the connections between PW and LCAO in TDDFT.

\section{Basic theory}

The macroscopic DF is given by a spatial average over the inverse microscopic DF [5]

\footnotetext{
* Corresponding author at: Donostia International Physics Center (DIPC), Paseo
} Manuel de Lardizabal 4, E-20018 San Sebastián, Spain. $\epsilon(\boldsymbol{q}, \omega) \quad 1 / \epsilon_{\mathbf{G}_{0, \mathbf{G}^{\prime}}{ }_{0}}(\mathbf{q}, \omega)$.

The inverse microscopic DF is connected to the interacting response function $\chi_{G^{\prime \prime} G^{\prime}}(\mathbf{q}, \omega)$

$\epsilon_{\mathbf{G G}^{\prime}}^{1}(\mathbf{q}, \omega) \quad \delta_{\mathbf{G G}^{\prime}}+v_{\mathbf{G G}^{\prime \prime}} \chi_{\mathbf{G}^{\prime \prime} \mathbf{G}^{\prime}}(\mathbf{q}, \omega)$,

where $v_{\mathbf{G G}} \frac{4 \pi \delta_{G G^{\prime}}}{\mid \mathbf{G}+\boldsymbol{q}^{2}}$ is a Coulomb interaction matrix element between PWs. In the last equation and below in this text we assume summation over repeating indices unless they appear on right hand side of the equations. The interacting response function satisfies a Petersilka Gossman Gross equation [5,6]

$$
\chi_{\mathbf{G G}^{\prime}}(\mathbf{q}, \omega) \quad \chi_{\mathbf{G}^{\prime}}^{0}(\mathbf{q}, \omega)+\chi_{\mathbf{G G}^{\prime \prime}}^{0}(\mathbf{q}, \omega) K^{\mathbf{G}^{\prime \prime} \mathbf{G}^{\prime \prime \prime}}(\mathbf{q}) \chi_{\mathbf{G}^{\prime \prime \prime} \mathbf{G}^{\prime}}(\mathbf{q}, \omega) .
$$

Here the non interacting response function $\chi_{\mathbf{G G}^{\prime}}^{0}(\mathbf{q}, \omega)$ and the TDDFT interaction kernel $K^{\mathbf{G G}^{\prime}}(\mathbf{q})$ appear. In this work, we use the so called RPA approximation $K^{\mathbf{G G}^{\prime}}(\mathbf{q}) \quad v_{\mathbf{G G}^{\prime}}$ for the TDDFT kernel. The non interacting response function $\chi_{\mathbf{G G}^{\prime}}^{0}(\mathbf{q}, \omega)$ has a convenient expression in terms of the Kohn Sham (KS) eigenstates

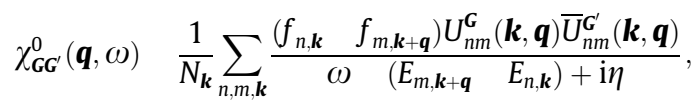

where the occupation numbers $f_{n, \boldsymbol{k}}$, band energies $E_{n, \boldsymbol{k}}$, broadening constant $\eta$ and number of $\boldsymbol{k}$ points $N_{\boldsymbol{k}}$ appear. $U_{n m}^{\boldsymbol{G}}(\boldsymbol{k}, \boldsymbol{q})$ are matrix elements of PWs in the basis of KS eigenstates $\Psi_{n, \boldsymbol{k}}(\boldsymbol{r})$

$U_{n m}^{G}(\boldsymbol{k}, \boldsymbol{q}) \equiv \frac{1}{\sqrt{ } V_{\mathrm{uc}}} \int_{V} \Psi_{n, \boldsymbol{k}}^{*}(\boldsymbol{r}) \mathrm{e}^{\mathrm{i}(\boldsymbol{q}+\boldsymbol{G}) \boldsymbol{r}} \Psi_{m, \boldsymbol{k}+\boldsymbol{q}}(\boldsymbol{r}) d^{3} r$. 


\section{Method}

Using the general expression for the macroscopic DF from Section 2, we can establish the connection to electronic structure calculations. We will start with LCAO eigenfunctions and expand the $D F(2)$ in a suitable set of localized basis functions.

\subsection{Localized orbitals in the bulk calculations}

We use LCAO in order to expand the eigenstates $\Psi_{n, \boldsymbol{k}}(\boldsymbol{r})$ of Kohn Sham Hamiltonian

$\Psi_{n, \boldsymbol{k}}(\boldsymbol{r}) \quad X_{a}^{n}(\boldsymbol{k}) \Phi^{a}(\boldsymbol{r}, \boldsymbol{k})$

in terms of Bloch symmetrized atomic orbitals [1,2]

$\Phi^{a}(\boldsymbol{r}, \boldsymbol{k}) \quad \frac{1}{\sqrt{ } N} \sum_{\boldsymbol{R}} \mathrm{e}^{\mathrm{i} \boldsymbol{k}\left(\boldsymbol{R}+\boldsymbol{R}_{a}\right)} f^{a}\left(\begin{array}{rll}\boldsymbol{r} & \boldsymbol{R}_{a} & \boldsymbol{R}) .\end{array}\right.$

Here the number of lattice translations $N$ enters in the normal ization constant (7). The summation in the last equation runs over lattice translations $\boldsymbol{R}$. The local atomic orbitals $f^{a}(\boldsymbol{r})$ are translated to each periodically repeated copy in the crystal. However, a finite spatial support of localized orbitals $f^{a}(\boldsymbol{r})$ leaves the possibility for operators to become sparse when the unit cell size exceeds the spatial support. The expansion coefficients in Eq. (6) $X_{a}^{n}(\boldsymbol{k})$, are determined during a self consistent Kohn Sham procedure. We use a DFT package SIESTA [12] for this step.

\subsection{Dominant products in the bulk calculations}

We want to use functions of finite support not only in DFT, but also in the TDDFT calculation of the DF (1). For this sake, we will construct a set of functions of finite support which is suitable for expanding the response functions (3) and (4). The matrix elements of PWs (5) contain the products of eigenstates. Therefore, it is obvi ous that the new product basis must represent these products accu rately. A product of eigenstates translates to a product of Bloch orbitals by virtue of the LCAO expansion (6). Using the definition of the Bloch orbital (7) one gets

$$
\bar{\Phi}^{a}(\boldsymbol{r}, \boldsymbol{k}) \Phi^{b}(\boldsymbol{r}, \boldsymbol{k}+\boldsymbol{q}) \quad \frac{1}{N} \sum_{\boldsymbol{R}, \boldsymbol{R}^{\prime}} \mathrm{e}^{\mathrm{i} \boldsymbol{k}\left(\boldsymbol{R}_{a}+\boldsymbol{R}\right)} \mathrm{e}^{\mathrm{i}(\boldsymbol{k}+\boldsymbol{q})\left(\boldsymbol{R}_{b}+\boldsymbol{R}^{\prime}\right)} \times
$$

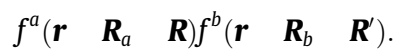

Due to the finite support of the local orbitals $f^{a}(\boldsymbol{r})$ and $f^{b}(\boldsymbol{r})$, the double infinite summation over lattice translations $\boldsymbol{R}$ and $\boldsymbol{R}^{\prime}$ can be converted to a summation in which only one translation runs infi nitely, while the other translation runs only in the neighborhood of the first. For this sake, we introduce a summation over translations $\boldsymbol{S} \quad \boldsymbol{R}^{\prime} \quad \boldsymbol{R}$ that will be finite. Using the super cell translation $\boldsymbol{S}$, one can rewrite Eq. (8)

$\bar{\Phi}^{a}(\boldsymbol{r}, \boldsymbol{k}) \Phi^{b}(\boldsymbol{r}, \boldsymbol{k}+\boldsymbol{q}) \quad \frac{1}{N} \sum_{\boldsymbol{R}, \boldsymbol{S}} \mathrm{e}^{\mathrm{i} \boldsymbol{k}\left(\boldsymbol{R}_{a}+\boldsymbol{R}\right)} \mathrm{e}^{\mathrm{i}(\boldsymbol{k}+\boldsymbol{q})\left(\boldsymbol{R}_{b}+\boldsymbol{R}+\boldsymbol{S}\right)} \times$

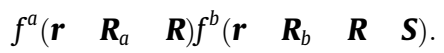

The product of localized function in the last equation is trans lated infinitely over the lattice. Hence, it is sufficient to find a rep resentation of this product for a zero translation $\boldsymbol{R} 0$. In our previous work we used a basis of dominant products $[13,14]$ to expand products of localized functions

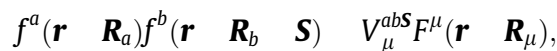

where expansion coefficients $V_{\mu}^{a b s}$ and product functions $F^{\mu}(\boldsymbol{r})$ are found in a diagonalization based procedure. The product functions $F^{\mu}\left(\boldsymbol{r} \quad \boldsymbol{R}_{\mu}\right)$ are centered at the midpoint of the connecting length
$\boldsymbol{R}_{\mu}\left(\boldsymbol{R}_{a}+\boldsymbol{R}_{b}+\boldsymbol{S}\right) / 2$. By inserting the ansatz (10) into Eq. (9) one gets

$\bar{\Phi}^{a}(\boldsymbol{r}, \boldsymbol{k}) \Phi^{b}(\boldsymbol{r}, \boldsymbol{k}+\boldsymbol{q}) \quad V_{\mu}^{a b}(\boldsymbol{k}, \boldsymbol{k}+\boldsymbol{q}) F^{\mu}(\boldsymbol{r}, \boldsymbol{q})$,

where a Bloch product vertex

$V_{\mu}^{a b}(\boldsymbol{k}, \boldsymbol{k}+\boldsymbol{q}) \quad \mathrm{e}^{\mathrm{i} \boldsymbol{k} \boldsymbol{R}_{a}} \mathrm{e}^{\mathrm{i} \boldsymbol{q} \boldsymbol{R}_{\mu}} \sum_{\boldsymbol{S}} V_{\mu}^{a b \boldsymbol{S}} \mathrm{e}^{\mathrm{i}(\boldsymbol{k}+\boldsymbol{q})\left(\boldsymbol{R}_{b}+\boldsymbol{S}\right)}$,

and a Bloch dominant product function are used

$F^{\mu}(\boldsymbol{r}, \boldsymbol{q}) \quad \frac{1}{N} \sum_{\boldsymbol{R}} \mathrm{e}^{\mathrm{i} \boldsymbol{q}\left(\boldsymbol{R}_{\mu}+\boldsymbol{R}\right)} F^{\mu}\left(\boldsymbol{r} \quad \boldsymbol{R}_{\mu} \quad \boldsymbol{R}\right)$.

Finally, there is a possibility of using only atom centered func tions in the ansatz (11) instead of using functions centered on the midpoint of two atoms. We will use these atom centered products in the calculations, although we have to skip a formal derivation here.

\subsection{Expansion of response function}

The expansion (11) can be used in the non interacting response function (4). To this end, we insert the product of Bloch orbitals (11) with help of Eq. (6) into the matrix element (5)

$U_{n m}^{\boldsymbol{G}}(\boldsymbol{k}, \boldsymbol{q}) \quad \bar{X}_{a}^{n}(\boldsymbol{k}) V_{\mu}^{a b}(\boldsymbol{k}, \boldsymbol{k}+\boldsymbol{q}) X_{b}^{m}(\boldsymbol{k}+\boldsymbol{q}) F_{G}^{\mu}(\mathbf{q})$,

where the Fourier transform of the Bloch function (12) appears

$F_{\boldsymbol{G}}^{\mu}(\boldsymbol{q}) \quad \frac{1}{\sqrt{ } V_{\mathrm{uc}}} \mathrm{e}^{\mathrm{i} \boldsymbol{G} \boldsymbol{R}_{\mu}} \int_{V} \mathrm{e}^{\mathrm{i}(\boldsymbol{q}+\boldsymbol{G}) \boldsymbol{r}} F^{\mu}(\boldsymbol{r}) d^{3} r$.

Here $V_{\mathrm{uc}}$ is the volume of unit cell. Using Eq. (13), we find for the response function (4)

$\chi_{\mathbf{G G}^{\prime}}^{0}(\mathbf{q}, \omega) \quad F_{\boldsymbol{G}}^{\mu}(\mathbf{q}) \chi_{\mu \nu}^{0}(\mathbf{q}, \omega) \bar{F}_{\boldsymbol{G}}^{v}(\mathbf{q})$.

Here the expansion coefficients $\chi_{\mu v}^{0}(\mathbf{q}, \omega)$ must be defined by

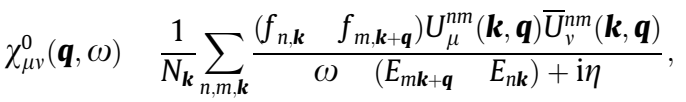

where the expansion coefficients $U_{\mu}^{n m}(\boldsymbol{k}, \boldsymbol{q})$ of products of eigenstates in terms of product functions are used

$U_{\mu}^{n m}(\boldsymbol{k}, \boldsymbol{q}) \quad \bar{X}_{a}^{n}(\boldsymbol{k}) V_{\mu}^{a b}(\boldsymbol{k}, \boldsymbol{k}+\boldsymbol{q}) X_{b}^{m}(\boldsymbol{k}+\boldsymbol{q})$.

Furthermore, for the interacting response function $\chi_{\mathbf{G} \mathbf{G}^{\prime}}(\mathbf{q}, \omega)$ we use an ansatz similar to Eq. (14) and rewrite Eq. (3) in the basis of Bloch product functions

$\chi_{\mu v}(\mathbf{q}, \omega) \quad \chi_{\mu v}^{0}(\mathbf{q}, \omega)+\chi_{\mu \mu^{\prime}}^{0}(\mathbf{q}, \omega) K^{\mu^{\prime} v^{\prime}}(\mathbf{q}) \chi_{v^{\prime} v}(\mathbf{q}, \omega)$,

where the interaction kernel $K^{\mu v}(\boldsymbol{q})$ has to be introduced

$K^{\mu v}(\boldsymbol{q}) \quad \sum_{\mathbf{G} \mathbf{G}^{\prime}} \bar{F}_{\mathbf{G}}^{\mu}(\boldsymbol{q}) K^{\mathbf{G G}^{\prime}}(\boldsymbol{q}) F_{\mathbf{G}^{\prime}}^{v}(\boldsymbol{q})$.

In the calculations, we only use the Hartree kernel $K^{\mathbf{G G}^{\prime}}(\boldsymbol{q}) \quad v_{\mathbf{G G}^{\prime}}(\boldsymbol{q})$, although any local or semi local interaction ker nel can be used.

\subsection{Iterative computation of dielectric function}

Using Eqs. (1), (2) with ansatz (14), we get the inverse macro scopic DF in terms of localized functions

$\epsilon^{1}(\boldsymbol{q}, \omega) \quad 1+\frac{4 \pi}{q^{2}} F_{\mathbf{0}}^{\mu}(\mathbf{q}) \chi_{\mu \nu}(\mathbf{q}, \omega) \bar{F}_{\mathbf{0}}^{v}(\mathbf{q})$,

where the interacting response function $\chi_{\mu v}(\mathbf{q}, \omega)$ can be deter mined by solving Eq. (16). The solution of the matrix equation is a 
computationally demanding task. However, if only the DF (18) is needed, then one can avoid the solution of matrix Eq. (16). We reformulate the problem in terms of solving of a linear equation

\section{$\left(1 \quad \chi^{0} K\right) X \quad \chi^{0} \bar{F}_{0}$}

and a subsequent calculation of the DF (18) $\epsilon^{1}(\boldsymbol{q}, \omega) \quad 1+\frac{4 \pi}{q^{2}} F_{0}^{\mu}(\boldsymbol{q}) X_{\mu}(\boldsymbol{q})$. Moreover, applying an iterative, Krylov subspace scheme [15] for solving the linear equation, one can signif icantly reduce the computational cost. The whole problem of com putation of the DF will be done in terms of matrix vector operations, applying $\left(1 \chi^{0} K\right)_{\mu}^{v}$ to vectors $z_{v}$. One can split the matrix vector operation in two: application of the kernel $K$ to the vector $z_{v}$ and subsequent application of response function to the intermediate vector $K z$. The kernel (17) is a full matrix. We pre compute the kernel before the iterative procedure and apply it to vectors using standard BLAS subroutines. The application of the non interacting response function (15) to a vector can also be split into several steps. The particular sequence of operations in the com putation of $\chi_{\mu \nu}^{0} z^{v}$ is shown in Fig. 1. Firstly, we compute the product $V_{v}^{c d} z^{v}$. Secondly, we multiply with the eigenvector $X_{d}^{n}$, etc. Because the vertex coefficients $V_{\mu}^{a b}$ are $\boldsymbol{k}$ dependent, we perform the sum over the Brillouin zone (BZ) in an outer loop and compute products $\chi_{\mu v}^{0} z^{v}$ simultaneously for a set of frequencies $\{\omega\}$. This method reduces the number of operations significantly. The asymptotical computation complexity of the method is $N_{a}^{3} N_{k}$, where $N_{a}$ is number of atoms in the unit cell. As iterative solver, we use modified CERF ACS subroutines which implement the Generalized Minimal Resi due method [16].

\section{Results}

In the Fig. 2, we compare the EELS of graphene computed by our iterative method with published calculations [6]. The parameters for this comparison were chosen as in the publication [6]. Namely, the momentum transfer $\boldsymbol{q} \quad 0.046 \AA^{1}$ in plane in the $\Gamma$ M direc tion, the distance between graphene layers $20 \AA$ and the sampling of BZ $64 \times 64 \times 1$ were used. The frequency broadening was set to $\eta \quad 0.24 \mathrm{eV}$. In the DFT calculation we used a double zeta polarized (DZP) basis with an energy shift parameter $50 \mathrm{meV}$, the Perdew Zunger (LDA) exchange correlation functional [17] and Troullier Martins pseudo potentials [18]. For the computation of the Hartree kernel (17), a set of PWs with an energy cutoff of $E_{\text {cut }} \quad 200 \mathrm{eV}$ (resulting in 651 PWs) was seen to give converged results and was subsequently used. The number of product basis functions was 138 (69 per atom in the unit cell), which is substantially lower than number of plane waves needed for convergence in this calcu lation. There are small discrepancies between the calculations. These discrepancies are most probably due to the basis sets used (spatial grid versus DZP basis in our calculation) as well as to the differences in the TDDFT kernel (LDA versus RPA).

The next comparison is shown in the Fig. 3. We compare our calculation for silicon with measurements [19] and with other independent calculations [20]. The parameters in the DFT calculation were the same as in the graphene example. We chose a small momentum transfer $q \quad 0.1336 \AA^{1}$, and an energy cutoff

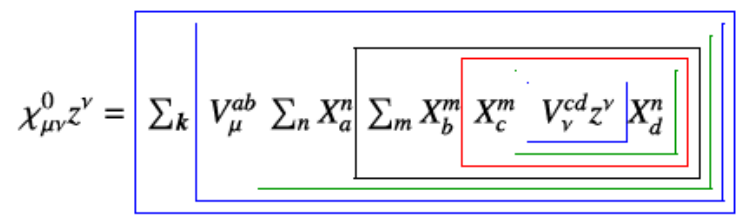

Fig. 1. The realized sequence of operations for $\chi^{0} \times$ vector product.

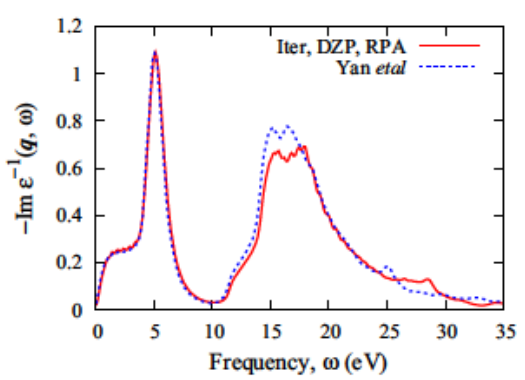

Fig. 2. EELS of graphene computed with our iterative method versus a mixed GridPW calculation [6].

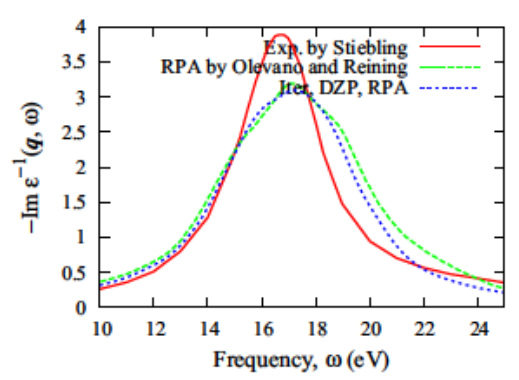

Fig. 3. EELS of silicon with computed with our iterative method versus calculation in PW basis [20] and measurements [19].

$E_{\text {cut }} 250 \mathrm{eV}$, which resulted in 339 PWs. The frequency broadening was set to $\eta \quad 0.6 \mathrm{eV}$. The $\mathrm{BZ}$ sampling was seen to be converged with $15 \times 15 \times 15$ points. The number of product basis functions was 136 ( 68 per atom in the unit cell), which is again lower than number of PWs needed for convergence (about 250). In Fig. 3 we see a good agreement between calculations but less satisfactory agreement with experiment. The discrepancies between theory and experiment must be attributed to deficiencies in the LDA functional and the RPA kernel.

The final calculation concerns the solid state of $C_{60}$ fullerenes. The structure and electronic properties of fullerite has been exten sively studied in the past [21 26]. It is known that buckminster fullerene crystallizes into one of the cubic lattices [21] and one should expect non negligible effects on the electronic structure induced by the crystal structure [27].

In this work, we compute EELS for the fcc crystal of $\mathrm{C}_{60}$ found by Dorset and McCourt [28]. The structure is a result of direct elec tron crystallographic analysis of crystals at room temperature most relevant for applications in organic electronics. The geometry was taken from an open crystallography database [29] (id 9011073) and converted to SIESTA format by cif2cell utility [30]. In the DFT calculation, we used the LDA functional, less extended atomic orbitals (energy shift parameter $200 \mathrm{meV}$ ), an electronic temperature of $300 \mathrm{~K}$ and a $3 \times 3 \times 3 \mathrm{BZ}$ sampling. The electronic structure corresponds to a semiconductor with a direct band gap of $0.85 \mathrm{eV}$. The gap value coincides rather well with other LDA calculation by Benning et al. [31] $(0.98 \mathrm{eV})$, a recent calcula tion by Zólyomi et al. [32] (1.06 eV) and with Troullier and Martins [33] $(1.18 \mathrm{eV})$. The momentum transfer was taken as the small value 0.0039 Bohr ${ }^{1}$ and the broadening constant was set to $0.6 \mathrm{eV}$. The $\mathrm{BZ}$ sampling was seen to be converged with $3 \times 3 \times 3$ points for the resolution defined by broadening of $\eta \quad 0.6 \mathrm{eV}$. An energy cutoff $E_{\text {cut }} \quad 200 \mathrm{eV}$ was used to define the set of PWs, which resulted in 4015 PWs. The number of product basis func tions was 4140 ( 69 functions per atom, i.e. the same as in the case of graphene example). The runtime on a 12 core machine with Intel Xeon X5550 processor at $2.67 \mathrm{GHz}$ was $10 \mathrm{~h}$, during which a 


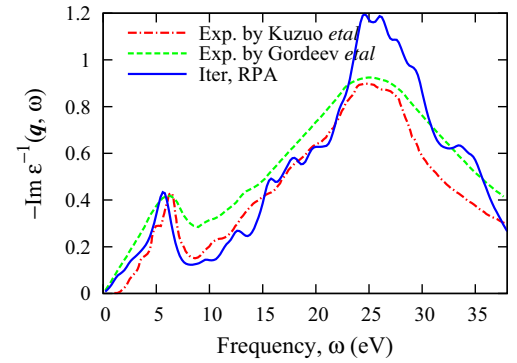

Fig. 4. EELS of solid $C_{60}$ computed with our iterative method is compared to measured spectra. Experimental data is taken from $[23,26]$.

maximum of 10 GMRES iterations per frequency point was performed. EELS curves computed with 9 and 10 iterations are not distinguishable on the plot. In Fig. 4 we see a comparison of our calculation with experimental data taken from $[23,26]$. As in the case of other carbon only systems, we find a low frequency $\pi \quad \pi^{*}$ resonance (at about $5.6 \mathrm{eV}$ in the calculation and at about $6.4 \mathrm{eV}$ in the measurements) and $\sigma \pi$ resonance (at about $25 \mathrm{eV}$ in calculation and measurements). At low resolution, the theoretical result match experimental data rather well. However, a more detailed comparison with higher resolution data in the low frequency range $\left(\begin{array}{ll}0 & 12 \mathrm{eV}\end{array}\right)$ is less satisfactory. The discrepan cies are probably connected to the accuracy of the DFT functional (determining $\chi^{0}$ ) and the TDDFT kernel (RPA in our case). However, the method presented here opens a possibility of practical TDDFT calculations for large systems which would be difficult to achieve with other methods.

\section{Acknowledgments}

We acknowledge support from ANR OrgaVolt project. D.S P. and P.K. acknowledge support from the Basque Departamento de
Educación, UPV/EHU (Grant No. IT 366 07), the Spanish Ministerio de Ciencia e Innovación (Grant No. FIS2010 19609 C02 02), the ETORTEK program funded by the Basque Departamento de Indu stria and the Diputación Foral de Guipuzcoa. D.S P., P.K. and M.P.L. acknowledge the German DFG through SFB 1083.

\section{References}

[1] C. Kittel, Introduction to Solid State Physics, John Wiley \& Sons Inc, New York, 1986.

[2] R.M. Martin, Electronic Structure: Basic Theory and Practical Methods, Cambridge University Press, Cambridge, 2004

[3] F.J. García de Abajo, Rev. Mod. Phys. 82 (2010) 209.

[4] A. Marini et al., Comput. Phys. Commun. 180 (2009) 1392.

[5] F. Sottile et al., Int. J. Quant. Chem. 102 (2005) 684.

[6] J. Yan et al., Phys. Rev. B 83 (2011) 245122.

[7] H. Jiang et al., Comput. Phys. Commun. 184 (2013) 348.

[8] X. Gonze et al., Comput. Phys. Commun. 180 (2009) 2582.

[9] A.F. Izmaylov, G.E. Scuseria, Phys. Rev. B 77 (2008) 165131.

[10] M. Rohlfing et al., Phys. Rev. B 52 (1995) 1905.

[11] F. Kootstra et al., J. Chem. Phys. 112 (2000) 6517.

[12] J.M. Soler et al., J. Phys. Condens. Matter 14 (2002) 2745.

[13] D. Foerster, J. Chem. Phys. 128 (2008) 034108.

[14] D. Foerster, P. Koval, J. Chem. Phys. 131 (2009) 044103.

[15] Y. Saad, Iterative methods for sparse linear systems, SIAM (2003).

[16] V. Frayssé et al., ACM Trans. Math. Softw. 35 (2008) 1.

[17] J.P. Perdew, A. Zunger, Phys. Rev. B 23 (1981) 5048.

[18] N. Troullier, J.L. Martins, Phys. Rev. B 43 (1991) 1993.

[19] J. Stiebling, Z. Phys, B 31 (1978) 355.

[20] V. Olevano, L. Reining, Phys. Rev. Lett. 86 (2001) 5962.

[21] W.I.F. David et al., Europhys. Lett. 18 (1992) 219.

[22] P. Wochner et al., Phys. Rev. B 55 (1997) 5678.

[23] R. Kuzuo et al., Jpn. J. Appl. Phys. 30 (1991) L1817.

[24] E. Sohmen, J. Fink, Phys. Rev. B 47 (1993) 14532.

[25] R. Kuzuo et al., Phys. Rev. B 49 (1994) 5054.

[26] Y. Gordeev et al., Phys. Solid State 42 (2000) 381.

[27] M.P. Gelfand, J.P. Lu, Phys. Rev. Lett. 68 (1992) 1050.

[28] D.L. Dorset, M.P. McCourt, Acta Cryst. A 50 (1994) 344.

[29] S. Gražulis et al., Nucleic Acids Res. 40 (2012) D420.

[30] B. Torbjörn, Comput. Phys. Commun. 182 (2011) 1183.

[31] P.J. Benning et al., Science 252 (1991) 1417.

[32] V. Zólyomi et al., Phys. Rev. B 78 (2008) 115405.

[33] N. Troullier, J.L. Martins, Phys. Rev. B 46 (1992) 1754 